\title{
LOCAL TO GLOBAL THEOREMS IN THE THEORY OF HUREWICZ FIBRATIONS
}

\author{
BY \\ JAMES E. ARNOLD, JR.
}

\begin{abstract}
This paper is concerned with the problem of showing a local fibration is a fibration. There are two kinds of local to global theorems proven. The first type of theorem considers local fibrations where local is in terms of closed covers of the base (e.g. the set of closed simplices of a polyhedron, the cones of a suspension). The second type of theorem deals with local in terms of open covers of the total space.
\end{abstract}

1. Introduction. The now classical Uniformization Theorem in the theory of fibrations [1] states that in the paracompact situation a local fibration is a fibration, where local is in terms of an open cover of the base. One of the main objectives of this paper is to derive similar local to global theorems in cases where the covers are closed, e.g. the set of closed simplices of a polyhedron. In addition, we also prove (with more generous hypothesis) the Hurewicz fibrations version of a result of Cheeger and Kister [2], that a local local disk bundle is a bundle.

2. Preliminary definitions and notation. Given a topological space $X$ we will be studying the category $\mathscr{F}(X)$ of spaces over $X$. An object in $\mathscr{F}(X)$ is a triple $\xi=(E, p, X)$ where $p: E \rightarrow X$ is a map. If $\xi=(E, p, X)$ and $\xi^{\prime}=\left(E^{\prime}, p^{\prime}, X\right)$ are two spaces over $X$, a morphism $f: \xi \rightarrow \xi^{\prime}$ in this category is a fiber preserving map $f: E \rightarrow E^{\prime}$, i.e. $p^{\prime} f(e)=p(e)$ for all $e \in E$. If $A \subset X$ and $\xi=(E, p, X)$ we will denote by $\xi_{A}=\left(E_{A}, p_{A}, A\right)$ the triple $\left(p^{-1}(A), p \mid p^{-1}(A), A\right)$ in $\mathscr{F}(A)$. Often we will use $p$ for the restriction $p_{A}$ when there is no chance for confusion. Furthermore, the notation $E_{A}$ does not exhibit the map $p$ as it should. However, the text will make clear the map in question used to form $E_{A}$.

An object $\xi=(E, p, X)$ in $\mathscr{F}(X)$ will be called a Hurewicz fibration if it has the universal covering homotopy property (CHP), or equivalently there is a lifting function $\lambda: \Omega_{p} \rightarrow E^{I}$. Recall that a lifting function for $\xi=(E, p, X)$ is a map

such that

$$
\lambda: \Omega_{p}=\left\{(e, \omega) \in E \times X^{I} \mid p(e)=\omega(0)\right\} \rightarrow E^{I}
$$

$$
\lambda(e, \omega)(0)=e \text { and } p \lambda(e, \omega)(t)=\omega(t) .
$$

A Hurewicz fibration will be called regular if it has a regular lifting function, that is, a lifting function such that if $\omega$ is a constant path, $\lambda(e, \omega)$ is a constant path for any $e$ with $(e, \omega) \in \Omega_{p}$.

Received by the editors November 18, 1970.

AMS 1970 subject classifications. Primary 55F05; Secondary 55F15, 55F10, 55F65.

Key words and phrases. Hurewicz fibrations, local to global, lifting functions, Lifting Extension Theorem, local local fibrations, Serre fibrations, Uniformization Theorem. 
Definition 2.1. $A \subset X$ (or the pair $(X, A)$ ) is called a cofibration if, for any space $Y$, any partial homotopy $H: X \times\{0\} \cup A \times I \rightarrow Y$ can be extended to a homotopy $\bar{H}: X \times I \rightarrow Y$.

Classically this property was called the homotopy extension property (HEP), and in Theorem 3.5 this terminology is more appropriate.

We will say $(X, A)$ is a closed cofibration if in addition $A$ is closed. If $X$ is a Hausdorff space $A \subset X$, a cofibration $\Rightarrow A$ is closed, so for a reasonable class of spaces, this is no real restriction.

DEFINITION 2.2. $X$ is a uniformly locally contractible (ULC) space if there is an open set $U \subset X \times X$ containing the diagonal $\Delta X$ of $X$ and a map $\sigma: U \rightarrow X^{I}$ with

(i) $\sigma(x, y)(0)=x$,

(ii) $\sigma(x, y)(1)=y$,

(iii) $\sigma(x, x)(t)=x$, all $t \in I$.

REMARK. If $\Delta X \subset X \times X$ is a closed cofibration, then $X$ is ULC.

In fact if $X$ is metric, then $X$ is ULC if and only if ${ }_{\Delta} X \subset X \times X$ is a cofibration.

This is a useful category of spaces since it includes the ANR's. Whether it is a bigger category is still unknown in the infinite-dimensional case.

Finally, given a path $\omega: I \rightarrow X$ and $s \in I$ we will have occasion to use the paths $\omega_{s}$ and $\omega^{s}$ defined as follows:

$$
\begin{aligned}
\omega^{s}(t) & =\omega(t+s) & & \text { if } 0 \leqq t+s \leqq 1 \\
& =\omega(1) & & \text { otherwise } \\
\omega_{s}(t) & =\omega(t) & & \text { if } 0 \leqq t \leqq s \\
& =\omega(s) & & \text { if } s \leqq t \leqq 1
\end{aligned}
$$

3. Cofibrations and fibrations. We will need the following two lemmas, proofs for which can be found in [3].

LemMA 3.1. If $(X, A),(Y, B)$ are closed cofibrations, then $(X \times Y, X \times B \cup A \times Y)$ is a closed cofibration.

LeMmA 3.2. If $(X, A)$ is a closed cofibration and $\xi=(E, p, X)$ is a Hurewicz fibration, then $E_{A} \subset E$ is a closed cofibration.

Lemma 3.3. If $\xi=(E, p, X)$ is a Hurewicz fibration and $(X, A)$ is a closed cofibration, then $\left(\Omega_{p}, \Omega_{p_{A}}\right)$ is a closed cofibration.

Proof. Let $\pi: \Omega_{p} \rightarrow X^{I}$ by $\pi(e, \omega)=\omega$. Then $\left(\Omega_{p}, \pi, X^{I}\right)$ is a Hurewicz fibration since it is the pullback of $\xi$ by the map $\alpha: X^{I} \rightarrow X$ which takes $\omega \rightarrow \omega(0)$. The result then follows from Lemma 3.2 since $A \subset X$ a closed cofibration $\Rightarrow A^{I} \subset X^{I}$ is a closed cofibration.

For fibrations we have the notion of Fiber Homotopy Extension Property (FHEP) which corresponds to the Homotopy Extension Property (HEP).

Definition 3.4. $(X, A)$ has the FHEP if and only if for any Hurewicz fibration $(E, p, B)$ and map $G: X \times\{0\} \cup A \times I \rightarrow E$ such that $p G(x, t)=p G(x, 0), x \in A$, $0 \leqq t \leqq 1$, there is an extension $H: X \times I \rightarrow E$ of $G$ such that $p H(x, t)=p H(x, 0)$. 
The following theorem is a reformulation of a result of Allaud and Fadell [4]. We will include a proof here since the proof in [4] does not cover the case of a Hurewicz fibration without a regular lifting function.

Theorem 3.5. $(X, A), A$ closed, has the FHEP if and only $(X, A)$ has the HEP.

Proof. If $(X, A)$ has the HEP we have a retraction $r: X \times I \rightarrow X \times\{0\} \cup A \times I$. We define $\Phi: X \rightarrow I$ by

$$
\Phi(x)=\max _{t \in I}\left|\pi_{2} r(x, t)-t\right| .
$$

Here $\pi_{2}: X \times\{0\} \cup A \times I \rightarrow I$ is projection on the second coordinate. Using the assumption that $A$ is closed it is easy to see that $\Phi(x)=0$ if and only if $x \in A$.

Given $\xi=(E, p, B)$ a Hurewicz fibration and $G: X \times\{0\} \cup A \times I \rightarrow E$ as above, $G$ can be extended to $X \times I$ by taking $G r: X \times I \rightarrow E$. We now define a map $F: I \times X \rightarrow B^{I}$ by

$$
\begin{aligned}
F(t, x)(s) & =p G r\left(x, \frac{\Phi(x) \cdot t-s(2-\Phi(x))}{\Phi(x)}\right) \quad \text { if } 0 \leqq s \leqq \Phi(x) \cdot t / 2 \text { and } \Phi(x) \neq 0, \\
& =p G r(x, t) \text { if } 0 \leqq s \leqq \Phi(x) \cdot t / 2 \text { and } \Phi(x)=0 \\
& =p G r(x, \Phi(x) \cdot t-s) \text { if } \Phi(x) \cdot t / 2 \leqq s \leqq \Phi(x) \cdot t \\
& =p G r(x, 0) \quad \text { if } \Phi(x) \cdot t \leqq s \leqq 1
\end{aligned}
$$

If we let

$$
H(x, t)=\lambda(G r(x, t), F(t, x))(\Phi(x) \cdot t)
$$

where $\lambda$ is a lifting function for $\xi, H$ satisfies the requirements of Definition 3.4.

Conversely, if $(X, A)$ has the FHEP and we have a map $h: X \times\{0\} \cup A \times I \rightarrow Y$ we wish to extend, let $p: Y \rightarrow B$ where $B$ is a point. Then we can apply the FHEP to $h$ and the Hurewicz fibration $(Y, p, B)$ to obtain the desired extension.

4. Some local to global theorems. Using the Fiber Homotopy Extension Theorem (Theorem 3.5) we now establish the Lifting Extension Theorem which will allow us to match lifting functions over cofibrations.

THEOREM 4.1. If $(X, A)$ is a closed cofibration and $\xi=(E, p, X)$ a Hurewicz fibration, then any lifting function $\lambda_{A}$ for $\xi_{A}$ extends to a lifting function for $\xi$.

Proof. By Lemma 3.3, $\Omega_{p_{A}} \subset \Omega_{p}$ is a closed cofibration. Therefore by Lemma 3.1, $\left(\Omega_{p} \times I, \Omega_{p} \times\{0\} \cup \Omega_{p_{A}} \times I\right)$ is a closed cofibration and has the FHEP by Theorem 3.5. Let $\lambda$ be a lifting function for $\xi$ and define $G: \Omega_{p} \times I \times\{0\} \cup \Omega_{p} \times\{0\} \times I$ $\cup \Omega_{p_{A}} \times I \times I \rightarrow E$ as follows:

$$
\begin{aligned}
G((e, \omega), t, s) & =\lambda(e, \omega)(t) & & \text { if } s=0,(e, \omega) \in \Omega_{p}, \\
& =e & & \text { if } t=0,(e, \omega) \in \Omega_{p}, \\
& =\lambda_{A}(e, \omega)(t) & & \text { if } 0 \leqq t \leqq s,(e, \omega) \in \Omega_{p_{A}} \\
& =\lambda\left[\lambda_{A}(e, \omega)(s), \omega^{s}\right](t-s) & & \text { if } s \leqq t \leqq 1,(e, \omega) \in \Omega_{p_{A}}
\end{aligned}
$$


Now since $\left(\Omega_{p} \times I, \Omega_{p} \times\{0\} \cup \Omega_{p_{A}} \times I\right)$ has the FHEP there is a map $H: \Omega_{p} \times I \times I \rightarrow E$ extending $G$, such that $p H((e, \omega), t, s)=p H((e, \omega), t, 0)$. Thus

$$
p H((e, \omega), t, s)=p G((e, \omega), t, 0)=\omega(t) .
$$

Therefore if we let $\lambda: \Omega_{p} \rightarrow E^{I}$ be $\lambda(e, \omega)(t)=H((e, \omega), t, 1), \lambda$ will be the desired lifting function for $\xi$ extending $\lambda_{A}$.

We can now prove some local to global theorems.

THEOREM 4.2. Let $X$ be a metric space of the form $X=X_{1} \cup X_{2}$ with $X_{1} \cap X_{2}$ $\subset X_{i}$ a cofibration, and $X_{i} U L C, i=1,2$. Then if $\xi=(E, p, X)$ is such that $\xi_{X_{1}}$ and $\xi_{X_{2}}$ are Hurewicz fibrations, $\xi$ is a Hurewicz fibration.

REMARK. Note that $X_{1}$ and $X_{2}$ are closed sets in this theorem.

Proof. By the Lifting Extension Theorem we can assume there are lifting functions $\lambda_{1}$ and $\lambda_{2}$ for $\xi_{X_{1}}$ and $\xi_{X_{2}}$ respectively such that $\lambda_{1}$ and $\lambda_{2}$ agree as lifting functions for $\xi_{X_{1} \cap X_{2}}$. Furthermore, since $X$ is metric, we can assume $\lambda_{1}$ and $\lambda_{2}$ are regular. By the Uniformization Theorem [1], we need only show that every point in $X$ has a neighborhood $U$ such that $\xi_{U}$ is a Hurewicz fibration, and obviously it is sufficient to consider points in $X_{1} \cap X_{2}$ every neighborhood of which intersects $X_{1}-X_{1} \cap X_{2}$ and $X_{2}-X_{1} \cap X_{2}$ nontrivially.

Since $X_{1} \cap X_{2} \subset X_{i}$ are cofibrations, $X_{1} \cap X_{2}$ is a neighborhood retract of $X$. Therefore by West [5, Lemma 5.8], given $x_{0} \in X_{1} \cap X_{2}$, there is a neighborhood $U$ of $x_{0}$ and a map $\sigma: U \times U \rightarrow X^{I}$ such that

(1) $\sigma(x, y)(0)=x$,

(2) $\sigma(x, y)(1)=y$,

(3) $\sigma(x, x)(t)=x$, all $t \in I$.

(4) If $x \in U_{i}=X_{i} \cap U$ then $\sigma(x, y)(t) \in X_{i}$ for $0 \leqq t \leqq \frac{1}{2}, i=1,2$.

(5) If $y \in U_{i}$ then $\sigma(x, y)(t) \in X_{i}$ for $\frac{1}{2} \leqq t \leqq 1, i=1,2$.

We can now define a slicing function $\psi: E_{U} \times U \rightarrow E_{U}$ as follows:

$$
\begin{aligned}
& \psi(e, x)=\lambda_{1}\left[\lambda_{1}\left(e, \sigma(p(e), x)_{1 / 2}\right)(1), \sigma(p(e), x)^{1 / 2}\right](1) \quad \text { if }(e, x) \in E_{U_{1}} \times U_{1}, \\
& =\lambda_{2}\left[\lambda_{2}\left(e, \sigma(p(e), x)_{1 / 2}\right)(1), \sigma(p(e), x)^{1 / 2}\right](1) \quad \text { if }(e, x) \in E_{U_{2}} \times U_{2} \text {, } \\
& =\lambda_{2}\left[\lambda_{1}\left(e, \sigma(p(e), x)_{1 / 2}\right)(1), \sigma(p(e), x)^{1 / 2}\right](1) \quad \text { if }(e, x) \in E_{U_{1}} \times U_{2} \text {, } \\
& =\lambda_{1}\left[\lambda_{2}\left(e, \sigma(p(e), x)_{1 / 2}\right)(1), \sigma(p(e), x)^{1 / 2}\right](1) \quad \text { if }(e, x) \in E_{U_{2}} \times U_{1} \text {. }
\end{aligned}
$$

$\psi$ is a slicing function, that is, $p \psi(e, x)=x$ and $\psi(e, p(e))=e$, and thus $\xi_{U}$ is a Hurewicz fibration. (A lifting function $\lambda$ is defined by $\lambda(e, \omega)(t)=\psi(e, \omega(t))$.) Therefore by the Uniformization Theorem, $\xi$ is a Hurewicz fibration.

COROLlaRY 4.3. If $K$ is a locally finite simplicial complex and $\xi=(E, p,|K|)$ is such that $\xi_{\delta^{n}}$ is a Hurewicz fibration for each closed simplex $\bar{\sigma}^{n}, \sigma^{n} \in K$, then $\xi$ is a Hurewicz fibration.

Proof. For finite simplicial complexes the proof is by induction on the number of closed simplices using the previous theorem for the inductive step. The general 
case follows from this since there is an open cover of $|K|,\left\{U_{\alpha}\right\}_{\alpha \in A}$ such that $\bar{U}_{\alpha}$ is a finite simplicial complex.

It is interesting to note that results similar to Theorem 4.2 and Corollary 4.3 hold in the locally trivial category. We will list them here without proof for comparison.

THEOREM 4.4. If $\xi=\left(E, p, X_{1} \cup X_{2}\right)$ is such that $\xi_{X_{1}}$ is locally trivial and $X_{1} \cap X_{2}$ $\subset X_{i}$ is a closed neighborhood retract, $i=1,2$, then $\xi$ is locally trivial.

CoROllaRY 4.5. If $K$ is a locally finite simplicial complex and $\xi=(E, p,|K|)$ is such that $\xi_{\sigma^{n}}$ is locally trivial for each closed simplex $\bar{\sigma}^{n}, \sigma^{n} \in K$, then $\xi$ is locally trivial.

5. Applications. The next theorem is an application of Corollary 4.3 to Serre fibrations. Recall that a triple $\xi=(E, p, X)$ is a Serre fibration if it has the CHP for polyhedra. This theorem generalizes the polyhedral case of a theorem of Ungar [6] which shows a Serre fibration with base and total space compact ANR's is a Hurewicz fibration.

TheOREM 5.1. Let $K$ and $L$ be simplicial complexes, $K$ locally finite. Then if $\xi=(|L|, p,|K|)$ is a Serre fibration, $\xi$ is a Hurewicz fibration.

Proof. By Corollary 4.3 it is sufficient to show that for any closed simplex $\bar{\sigma}^{n}, \sigma^{n} \in K, \xi_{\bar{\sigma}^{n}}$ is a Hurewicz fibration. Let $V$ be an open set containing $\bar{\sigma}^{n}$ such that there is a map $\alpha: V \times V \rightarrow|K|^{I}$ with

(i) $\alpha(x, y)(0)=x, x, y \in V$,

(ii) $\alpha(x, y)(1)=y, x, y \in V$,

(iii) $\alpha(x, x)(t)=x, x \in V, t \in I$.

By choosing a suitable subdivision $L^{\prime}$ of $L$ we can find a subcomplex $X$ of $L^{\prime}$ such that $p^{-1}(V) \supset|X| \supset p^{-1}\left(\bar{\sigma}^{n}\right)$. Now let $d:|K| \rightarrow I$ be a metric and consider the following diagram:

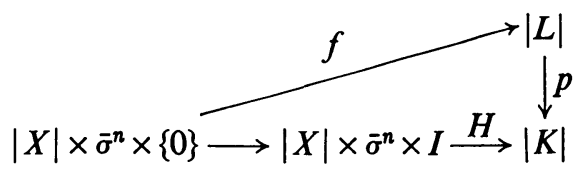

The maps $f$ and $H$ are given by $f(e, y)=e$ and

$$
\begin{aligned}
H(e, y, t) & =\alpha(p(e), y)(t / d(p(e), y)) & & \text { if } 0 \leqq t \leqq d(p(e), y) \neq 0, \\
& =p(e) & & \text { if } d(p(e), y)=0, \\
& =y & & \text { if } d(p(e), y) \leqq t \leqq 1 .
\end{aligned}
$$

Since $X$ is a simplicial complex and $\xi$ a Serre fibration, there is a map

with

$$
\bar{H}:|X| \times \bar{\sigma}^{n} \times I \rightarrow|L|
$$

(1) $\bar{H}(e, y, 0)=e$,

(2) $p \bar{H}(e, y, t)=H(e, y, t)$. 
We can now define a slicing function $\psi: p^{-1}\left(\bar{\sigma}^{n}\right) \times \bar{\sigma}^{n} \rightarrow p^{-1}\left(\bar{\sigma}^{n}\right)$ by

$$
\psi(e, x)=\bar{H}(e, x, d(p(e), x))
$$

Thus $\xi_{\bar{\sigma}^{n}}$ is a Hurewicz fibration and by Corollary 4.3, $\xi$ is a Hurewicz fibration.

The next theorem is an application of Theorem 4.2 which allows us to conclude that a map over a suspension is a Hurewicz fibration if and only if the part over each cone is a Hurewicz fibration.

THEOREM 5.2. Let $X$ be a metric space of the form $X=X_{1} \cup X_{2}$ with $X_{1} \cap X_{2}$ $\subset X_{i}$ a closed cofibration and $X_{i} U L C, i=1,2$. Then if $B$ is an arbitrary topological space and $\xi=(E, p, B \times X)$ is such that $\xi_{B \times X_{1}}$ is a Hurewicz fibration, $i=1,2, \xi$ is a Hurewicz fibration.

Proof. We will show that $\left(E, \pi_{1} p, B\right)$ and $\left(E, \pi_{2} p, X_{1} \cup X_{2}\right)$ are Hurewicz fibrations, and the lifting functions are nice enough so that we can put them together to obtain a lifting function for $\xi$. Here $\pi_{i}$ is again the projection of $B \times X$ into the $i$ th coordinate.

Let $\lambda_{i}$ be a lifting function for $\xi_{B \times X_{i}}, i=1,2$. Then, since $X_{1} \cap X_{2} \subset X_{1}$ is a closed cofibration, $B \times\left(X_{1} \cap X_{2}\right) \subset B \times X_{1}$ is also a closed cofibration and by the Lifting Extension Theorem we can assume $\lambda_{1}$ and $\lambda_{2}$ agree as lifting functions for $\xi_{B \times\left(X_{1} \cap X_{2}\right)}$. Now if $(e, \omega) \in \Omega_{\pi_{1} p}=\left\{(e, \omega) \in E \times B^{I} \mid \pi_{1} p(e)=\omega(0)\right\}$ let

$$
\begin{aligned}
\alpha_{1}(e, \omega)(t) & =\lambda_{1}\left[e,\left(\omega, \pi_{2} p(e)\right)\right](t) & & \text { if } e \in E_{B \times X_{1}}, \\
& =\lambda_{2}\left[e,\left(\omega, \pi_{2} p(e)\right)\right](t) & & \text { if } e \in E_{B \times X_{2}},
\end{aligned}
$$

where we consider $\left(\omega, \pi_{2} p(e)\right)$ as the path in $B \times X_{i}$ given by $\left(\omega, \pi_{2} p(e)\right)(t)$ $=\left(\omega(t), \pi_{2} p(e)\right)$. It is easy to check that $\alpha_{1}: \Omega_{\pi_{1} p} \rightarrow E^{I}$ is a lifting function for $\left(E, \pi_{1} p, B\right)$. Note also that $\pi_{2} p \alpha_{1}(e, \omega)(t)=\pi_{2} p(e)$. We will need this later when we define a lifting function for $\xi$.

Now consider $\left(E, \pi_{2} p, X_{1} \cup X_{2}\right)$. Lifting functions for $\left(E_{X_{i}}, \pi_{2} p, X_{i}\right), i=1,2$, are given by $\lambda_{i}(e, \omega)(t)=\lambda_{i}\left(e,\left(\pi_{1} p(e), \omega\right)\right)(t)$ where $\left(\pi_{1} p(e), \omega\right)$ is the path given by $\left(\pi_{1} p(e), \omega\right)(t)=\left(\pi_{1} p(e), \omega(t)\right)$. (Here $\left.E_{X_{i}}=\left(\pi_{2} p\right)^{-1}\left(X_{i}\right).\right)$ Note that

$$
\pi_{1} p \lambda_{i}(e, \omega)(t)=\pi_{1} p \lambda_{i}\left(e,\left(\pi_{1} p(e), \omega\right)\right)(t)=\pi_{1} p(e)
$$

and that $\lambda_{1}$ and $\lambda_{2}$ agree as lifting functions for $\left(E_{X_{1} \cap X_{2}}, \pi_{2} p, X_{1} \cap X_{2}\right)$.

The argument used to prove Theorem 4.2 will produce a regular lifting function $\alpha_{2}$ with the additional property that

$$
\pi_{1} p \alpha_{2}(e, \omega)(t)=\pi_{1} p(e)
$$

To see that (1) holds it is necessary to check that this property is preserved at each stage of the proof of Theorem 4.2 using $\lambda_{1}$ and $\lambda_{2}$. Since the argument is somewhat tedious, we will omit it here. 
Therefore we have a lifting function $\alpha_{1}$ for $\left(E, \pi_{1} p, B\right)$, and a regular lifting function $\alpha_{2}$ for $\left(E, \pi_{2} p, X\right)$ such that

$$
\pi_{1} p \alpha_{2}(e, \omega)(t)=\pi_{1} p(e)
$$

and

$$
\pi_{2} p \alpha_{1}(e, \omega)(t)=\pi_{2} p(e) .
$$

We now define a lifting function $\alpha_{1} \times \alpha_{2}$ for $(E, p, B \times X)$ by

$$
\alpha_{1} \times \alpha_{2}(e, \omega)(t)=\alpha_{1}\left[\alpha_{2}\left(e, \pi_{2} \omega_{t}\right)(1), \pi_{1} \omega\right](t) .
$$

The second lifting by $\alpha_{1}$ is defined since

$$
\pi_{1} p \alpha_{2}\left(e, \pi_{2} \omega_{t}\right)(1)=\pi_{1} p(e) \text { by }(1) \text {. }
$$

We can now see that $\alpha_{1} \times \alpha_{2}$ is a lifting function.

$$
\pi_{1} p\left(\alpha_{1} \times \alpha_{2}\right)(e, \omega)(t)=\pi_{1} p \alpha_{1}\left[\alpha_{2}\left(e, \pi_{2} \omega_{t}\right)(1), \pi_{1} \omega\right](t)=\pi_{1} \omega(t)
$$

since $\alpha_{1}$ is a lifting function for $\pi_{1} p$.

$$
\begin{aligned}
\pi_{2} p\left(\alpha_{1} \times \alpha_{2}\right)(e, \omega)(t) & =\pi_{2} p \alpha_{1}\left[\alpha_{2}\left(e, \pi_{2} \omega_{t}\right)(1), \pi_{1} \omega\right](t) \\
& =\pi_{2} p \alpha_{2}\left(e, \pi_{2} \omega_{t}\right)(1) \text { by }(2) \\
& =\pi_{2} \omega_{t}(1)=\pi_{2} \omega(t)
\end{aligned}
$$

since $\alpha_{2}$ is a lifting function for $\pi_{2} p$. Thus we have

$$
p\left(\alpha_{1} \times \alpha_{2}\right)(e, \omega)(t)=\left(\pi_{1} \omega(t), \pi_{2} \omega(t)\right)=\omega(t) .
$$

We need to show now that $\alpha_{1} \times \alpha_{2}(e, \omega)(0)=e$. If $t=0$ then $\omega_{t}=\omega_{0}$ is the constant path at $p(e)$ which implies

$$
\begin{aligned}
\alpha_{1} \times \alpha_{2}(e, \omega)(0) & =\alpha_{1}\left[\alpha_{2}\left(e, \pi_{2} \omega_{0}\right)(1), \pi_{1} \omega\right](0) \\
& =\alpha_{1}\left(e, \pi_{1} \omega\right)(0) \quad\left(\text { since } \alpha_{2} \text { is regular }\right) \\
& =e .
\end{aligned}
$$

Therefore $\xi=\left(E, p, B \times\left(X_{1} \cup X_{2}\right)\right)$ is a Hurewicz fibration.

For the following corollary we let $\Sigma X$ denote the suspension of $X$, i.e. $\Sigma X$ is $X \times[-1,1]$ with $X \times\{-1\}$ and $X \times\{1\}$ identified to points. Let $C+X$ be the "upper" cone in $\Sigma X$, that is, $X \times[0,1]$ with $X \times\{1\}$ identified to a point, and similarly $C-X$ is the "lower" cone.

Corollary 5.3. If $\xi=(E, p, \Sigma X)$ is such that $\xi_{C+X}$ and $\xi_{C-x}$ are Hurewicz fibrations, then $\xi$ is a Hurewicz fibration.

Proof. Consider $X \times\left[-\frac{1}{2}, \frac{1}{2}\right] \subset \Sigma X$. Then $\xi_{X \times[-1 / 2,0]}$ and $\xi_{X \times[0,1 / 2]}$ are Hurewicz fibrations. Therefore by Theorem 5.2, $\xi_{X \times[-1 / 2,1 / 2]}$ is a Hurewicz fibration. Now since $C+X, C-X$ and $X \times\left[-\frac{1}{2}, \frac{1}{2}\right]$ form a numerable cover of $\Sigma X$, it follows that $\xi$ is a Hurewicz fibration by the Uniformization Theorem (see [7]).

6. Local local theorems. In this section we will prove that we can localize the problem of deciding when a map is a Hurewicz fibration on the total space in much the same way as the Uniformization Theorem localizes the problem on the base. 
Definition 6.1. $\xi=(E, p, X)$ is locally locally a (regular) Hurewicz fibration if for any $e \in E$ there are neighborhoods $U$ of $e$ and $V$ of $p(e)$ such that $(U, p \mid U, V)$ is a (regular) Hurewicz fibration.

We will now list some technicalities as lemmas since they will be used many times in the proof of the main theorem of this section. We will omit the proofs since Lemmas 6.2 and 6.3 are well known, and Lemma 6.4 is a simple exercise using the definition of continuity and regularity of $\lambda$, and the compact open topology on $X^{I}$.

LemMA A 6.2. Suppose $(E, p, X)$ is such that $p$ is a proper map, $X$ and $E$ are Hausdorff, and $X$ is locally compact. Then for any open set $U \supset p^{-1}(x), x \in X$, there is a neighborhood $V$ of $x$ such that $p^{-1}(V) \subset U$.

Lemma B 6.3. Suppose $(E, p, X)$ has the property that $p$ is closed. Then for any open set $U \supset p^{-1}(x)$ there is a neighborhood $V$ of $x$ such that $p^{-1}(V) \subset U$.

Lemma $C$ 6.4. Suppose $\xi=(E, p, X)$ has a regular lifting function $\lambda$ and that $x \in X, e_{0} \in p^{-1}(x)$ and $U \subset E$ is a neighborhood of $e_{0}$. Then there are neighborhoods $V \subset X$ of $x$ and $U^{\prime} \subset p^{-1}(V)$ of $e_{0}$ with the following properties:

(1) $U^{\prime} \subset p^{-1}(V) \cap U$.

(2) If $\omega \in V^{I}, e \in U^{\prime}$ and $p(e)=\omega(0)$, then $\lambda(e, \omega)(t) \in U$, for all $t \in I$.

We can now prove a local local to global theorem.

TheOREM 6.5. Suppose $\xi=(E, p, X)$ is such that $X$ is paracompact and either

A. $p$ is proper, $X$ locally compact, and $E$ Hausdorff; or

B. $p$ is closed, $E$ metric, and $p^{-1}(x)$ compact for all $x \in X$.

Then if $\xi$ is locally locally a regular Hurewicz fibration, $\xi$ is a regular Hurewicz fibration.

Proof. We will prove the theorem under Hypothesis A. The proof under Hypothesis B is similar except that we use Lemma B instead of Lemma A, and use the metric on $E$ to define the functions $\Phi$ and $\psi$ used in the following proof for case A.

$X$ is paracompact, thus by the Uniformization Theorem we need only show that for any $x \in X$, there is a neighborhood $N$ of $x$ such that $\xi_{N}$ is a regular Hurewicz fibration. Since $p^{-1}(x)$ is compact and $\xi$ is locally locally a regular Hurewicz fibration there are open sets $U_{1}, \ldots, U_{n}$ and $V_{1}, \ldots, V_{n}$ such that $p^{-1}(x) \subset \bigcup_{i=1}^{n} U_{i}$ and $\left(U_{i}, p \mid U_{i}, V_{i}\right), i=1, \ldots, n$, are Hurewicz fibrations with regular lifting functions $\lambda_{i}, i=1, \ldots, n$, respectively. Also, since $\bigcup_{i=1}^{n} U_{i}$ is a neighborhood of $p^{-1}(x)$, we can, by Lemma $\mathrm{A}$, find a neighborhood $\hat{V}$ of $x$ such that $p^{-1}(\hat{V}) \subset \bigcup_{i=1}^{n} U_{i}$. Let $\hat{U}_{i}=p^{-1}(\hat{V}) \cap U_{i}$.

The data $\left\{\left\{\hat{U}_{i}\right\}_{i=1, \ldots, n} ; \hat{V}\right\}$ then has the following two properties:

(1) $\left(\hat{O}_{i}, p \mid \hat{O}_{i}, \hat{V}\right), i=1, \ldots, n$, are regular Hurewicz fibrations;

(2) $p^{-1}(\hat{V})=\bigcup_{i=1}^{n} \hat{U}_{i}$. 
The theorem will be proven by induction. We will show that given data $\left\{\left\{\hat{U}_{i}\right\}_{i=1, \ldots, n} ; \hat{V}\right\}$ as above we can find another set of data $\left\{\left\{\hat{U}_{i}^{\prime}\right\}_{i=1, \ldots, n-1} ; \hat{V}^{\prime}\right\}$ with the same properties.

Since $X$ is a locally compact Hausdorff space, there is a neighborhood $W$ of $x$ such that $\bar{W}$ is compact and $\bar{W} \subset \hat{V} \cdot p^{-1}(\bar{W})$ is a compact Hausdorff space, since $p$ is proper, and is the union of relatively open sets $Q_{i}=\hat{U}_{i} \cap p^{-1}(\bar{W})$ such that $\left(Q_{i}, p \mid Q_{i}, \bar{W}\right), i=1, \ldots, n$, are regular Hurewicz fibrations.

Consider $Q_{1}-\bigcup_{i=2}^{n} Q_{i}$ and $\bigcup_{i=2}^{n} Q_{i}-Q_{1}$. They are disjoint closed subsets of $\bigcup_{i=1}^{n} Q_{i}=p^{-1}(\bar{W})$, a normal space. Therefore we can find a Urysohn function $\Phi: \bigcup_{i=1}^{n} Q_{i} \rightarrow I$ with $\Phi\left(Q_{1}-\bigcup_{i=2}^{n} Q_{i}\right)=0$ and $\Phi\left(\bigcup_{i=2}^{n} Q_{i}-Q_{1}\right)=1$. $\Phi^{-1}\left[\frac{1}{4}, \frac{3}{4}\right]$ is a closed subset of $Q_{1} \cap \bigcup_{i=2}^{n} Q_{i}$ and thus $\Phi^{-1}\left[\frac{1}{4}, \frac{3}{4}\right] \cap p^{-1}(x)$ is compact. By Lemma $C$ we can cover $\Phi^{-1}\left[\frac{1}{4}, \frac{3}{4}\right] \cap p^{-1}(x)$ by a finite number of open sets $\tilde{Q}_{1}, \ldots, \tilde{Q}_{m}$ so that the lift of any path in $W \cap\left(\tilde{V}_{1} \cap \cdots \cap \tilde{V}_{m}\right)$ by $\lambda_{1}$ to any point in $\tilde{Q}_{1} \cup \cdots \cup \widetilde{Q}_{m}$ lies in $\Phi^{-1}\left(\frac{1}{8}, \frac{7}{8}\right)$. Here $\tilde{V}_{i}$ corresponds to $\tilde{Q}_{i}$ as in Lemma $\mathrm{C}$ and $\lambda_{1}$ is the fixed regular lifting function for $\left(U_{1}, p \mid U_{1}, V_{1}\right)$.

Now the set

$$
p^{-1}(W) \cap\left\{\Phi^{-1}\left[0, \frac{1}{4}\right) \cup \widetilde{Q}_{1} \cup \cdots \cup \widetilde{Q}_{m} \cup \Phi^{-1}\left(\frac{3}{4}, 1\right]\right\}
$$

is a neighborhood of $p^{-1}(x)$ in $p^{-1}(\bar{W})$. Therefore we can apply Lemma $\mathrm{A}$ to find a neighborhood $G$ of $x$ such that

$$
p^{-1}(G) \subset p^{-1}(W) \cap\left\{\Phi^{-1}\left[0, \frac{1}{4}\right) \cup \widetilde{Q}_{1} \cup \cdots \cup \tilde{Q}_{m} \cup \Phi^{-1}\left(\frac{3}{4}, 1\right]\right\} .
$$

Now let $L$ be a neighborhood of $x$ such that $\bar{L}$ is compact and $\bar{L} \subset G$. Then $p^{-1}(\bar{L})$ is a compact Hausdorff space. Let

$$
\begin{aligned}
Q_{i}^{*} & =Q_{i} \cap p^{-1}(\bar{L}) \text { and } \tilde{Q}_{i}^{*}=\widetilde{Q}_{i} \cap p^{-1}(\bar{L}), \\
A & =\Phi^{-1}\left[0, \frac{1}{4}\right) \cap p^{-1}(\bar{L})-\left(\widetilde{Q}_{1}^{*} \cup \cdots \cup \tilde{Q}_{m}^{*}\right),
\end{aligned}
$$

and

$$
B=\Phi^{-1}\left(\frac{3}{4}, 1\right] \cap p^{-1}(\bar{L})-\left(\widetilde{Q}_{1}^{*} \cup \cdots \cup \widetilde{Q}_{m}^{*}\right) .
$$

Then $A$ and $B$ are disjoint closed subsets of $p^{-1}(\bar{L})$. Now consider cl $\left(Q_{1}^{*} \cup Q_{2}^{*}\right) \cap A$ and $\operatorname{cl}\left(Q_{1}^{*} \cup Q_{2}^{*}\right) \cap B$. These are disjoint closed subsets of $\operatorname{cl}\left(Q_{1}^{*} \cup Q_{2}^{*}\right) \cdot \operatorname{cl}\left(Q_{1}^{*} \cup Q_{2}^{*}\right)$ is closed in $p^{-1}(\bar{L})$ and thus is a compact Hausdorff space. Therefore we can find another Urysohn function $\psi: \operatorname{cl}\left(Q_{1}^{*} \cup Q_{2}^{*}\right) \rightarrow I$ with

$$
\psi\left[\mathrm{cl}\left(Q_{1}^{*} \cup Q_{2}^{*}\right) \cap A\right]=1 \text { and } \psi\left[\operatorname{cl}\left(Q_{1}^{*} \cup Q_{2}^{*}\right) \cap B\right]=0 .
$$

We now define a regular lifting function for $Q_{1}^{*} \cup Q_{2}^{*}$ by

$$
\begin{aligned}
\lambda(e, \omega)(s) & =(1) \quad \lambda_{1}(e, \omega)(s) \text { if } e \in\left(Q_{1}^{*} \cup Q_{2}^{*}\right) \cap A, \\
& =(2) \quad \lambda_{2}(e, \omega)(s) \text { if } e \in\left(Q_{1}^{*} \cup Q_{2}^{*}\right) \cap B, \\
& =(3) \quad \lambda_{1}(e, \omega)(s) \text { if } 0 \leqq s \leqq \psi(e), \\
& =(4) \quad \lambda_{2}\left(\lambda_{1}(e, \omega)(\psi(e)), \omega^{\psi(e)}\right)(s-\psi(e)), \quad \text { if } \psi(e) \leqq s \leqq 1,
\end{aligned}
$$

where $\lambda_{2}$ is the regular lifting function chosen for $\left(U_{2}, p \mid U_{2}, V_{2}\right)$. 
Note that by the choice of the $\widetilde{Q}_{i}$ in the preceding argument the composition in part (4) is defined. It is clear that $\lambda$ is a regular lifting function for $Q_{1}^{*} \cup Q_{2}^{*}$. Therefore we define

$$
\begin{aligned}
& \hat{U}_{1}^{\prime}=\left(Q_{1}^{*} \cup Q_{2}^{*}\right) \cap p^{-1}(L), \\
& \hat{U}_{i}^{\prime}=Q_{i+1}^{*} \cap p^{-1}(L), \quad i=2, \ldots, n-1, \\
& \hat{V}^{\prime}=L .
\end{aligned}
$$

The data $\left\{\left\{\hat{U}_{i}^{\prime}\right\}_{i=1, \ldots, n-1} ; \hat{V}^{\prime}\right\}$ then has the following two properties:

(1) $\left(\hat{U}_{i}^{\prime}, p \mid \hat{U}_{i}^{\prime}, \hat{V}^{\prime}\right), i=1, \ldots, n-1$, are regular Hurewicz fibrations;

(2) $p^{-1}\left(\hat{V}^{\prime}\right)=\bigcup_{i=1}^{n-1} \hat{O}_{i}^{\prime}$.

By induction then, we can find a neighborhood $N$ of $x$ such that $\xi_{N}$ is a regular Hurewicz fibration, and thus since $X$ is paracompact, $\xi$ is a regular Hurewicz fibration.

\section{BIBLIOGRAPHY}

1. W. Hurewicz, On the concept of fiber space, Proc. Nat. Acad. Sci. U.S.A. 41 (1955), 956-961. MR 17, 519.

2. J. Cheeger and J. Kister, Counting topological manifolds, Topology 9 (1970), 149-151. MR 41 \#1055.

3. A. Strøm, Note on cofibrations. II, Math. Scand. 22 (1968), 130-142. MR 39 \#4846.

4. G. Allaud and E. Fadell, $A$ fiber homotopy extension theorem, Trans. Amer. Math. Soc. 104 (1962), 239-251. MR 30 \#4264.

5. D. C. West, I, On uniformly locally contractible spaces. II, Transitive translation functions in fibre bundles, Ph.D. Thesis, University of Wisconsin, Madison, Wis., 1965.

6. G. S. Ungar, Conditions for a mapping to have the slicing structure property, Pacific J. Math. 30 (1969), 549-553. MR 40 \#3550.

7. A. Dold, Partitions of unity in the theory of fibrations, Ann. of Math. (2) 78 (1963), 223-255. MR 27 \#5264.

Department of Mathematics, University of Wisconsin-Milwaukee, Milwaukee, WISCONSIN 53201 